\title{
Dynamic Characteristics and Key Parameter Optimization of Mechanical Automatic Vertical Drilling Tools
}

\author{
Jin Wang $\mathbb{D}^{1,2}$ Yuanbiao Hu $\mathbb{D}^{1,2}$ Zhijian Liu $\mathbb{D}^{3}{ }^{3}$ Lixin Li $\mathbb{D}^{4,5}$ Baolin Liu $\mathbb{D}^{1,2}$ \\ and Leilei Huang $\mathbb{C}^{1,2}$ \\ ${ }^{1}$ School of Engineering and Technology, China University of Geosciences, Beijing 100083, China \\ ${ }^{2}$ Key Laboratory of Deep Geodrilling Technology, Ministry of Land and Resources, Beijing 100083, China \\ ${ }^{3}$ Beijing Institute of Mineral Exploration Engineering, Beijing 100083, China \\ ${ }^{4}$ Chinese Academy of Geological Sciences, Beijing 100037, China \\ ${ }^{5}$ China Deep Exploration Center, China Geological Survey, Chinese Academy of Geological Sciences, Beijing 100037, China \\ Correspondence should be addressed to Yuanbiao Hu; hyb@cugb.edu.cn
}

Received 27 August 2020; Revised 18 December 2020; Accepted 8 January 2021; Published 23 January 2021

Academic Editor: Wenjun Huang

Copyright (C) 2021 Jin Wang et al. This is an open access article distributed under the Creative Commons Attribution License, which permits unrestricted use, distribution, and reproduction in any medium, provided the original work is properly cited.

\begin{abstract}
Mechanical automatic vertical drilling tools (MAVDT) have gradually gained attention as a drilling tool that can achieve active correction in harsh working environments such as high temperature and high pressure. The gravity sensing mechanism can sense the deviation and convert the deviation signal into the control signal to drive the actuator to correct the deviation. It is the core component of the mechanical automatic vertical drilling tool. This paper analyzes the force on the gravity sensing mechanism based on the structural analysis of the mechanical automatic vertical drilling tool. Then, the general dynamic equation of the gravity sensing mechanism is established based on D'Alembert principle. The critical response position where the acceleration value is zero is used as the research object to complete the preliminary design and analysis of the sensing mechanism. Through analysis, it can be found that there is a conflict between the response accuracy and control stability of the gravity sensing mechanism. High response accuracy often means poor control stability. For the gravity sensing mechanism with definite structural parameters, there is a limit value of the allowable friction coefficient. When the friction coefficient of the thrust bearing exceeds the limit value, the gravity sensing mechanism cannot achieve the inclination response no matter how big the inclination angle and deflection angle are. The friction coefficient between the disc valves and the force between the disc valves of the gravity sensing mechanism have a linear effect on the performance of the mechanism, and the smaller the deviation angle, the greater the influence coefficient of the force or the friction coefficient between the disc valves on the length of the gravity sensing mechanism. During the process of dynamic swing, the dynamic stable position of the gravity sensing mechanism is related to the relative relationship between the restoring force of the mechanism and the friction damping. To be precise, it is related to the potential energy zero point and the speed zero point during the gravity sensing mechanism swing process before it reaches the dynamic stable position.
\end{abstract}

\section{Introduction}

As one kind of active hole straightening tool, automatic vertical drilling tool (AVDT) is thought of as an advanced vertical drill technology, which changes the traditional well trajectory control method. This new rotary steerable system for vertical drilling can not only prevent boreholes from deviating from vertical but also correct inclined boreholes to verticality actively. Since the first application for the "Continental Deep Drilling Program" in 1990, the AVDT has obtained tremendous development and has been widely used in engineering. The Verti-Trak owned by Baker Hughes Incorporated and Power- $\mathrm{V}$ owned by Schlumberger Limited are typical representatives of them [1-4]. But, with the development of technology, the requirements for well depth are increasing, and there are more and more high temperature and high pressure wells in engineering. Due to the large number of electronic 
components, the traditional AVDT can no longer meet the needs of the operation [5-8].

Compared with the traditional electronically controlled AVDT, the mechanical automatic vertical drilling tool (MAVDT) can achieve continuous active correction under the action of gravity only through the pure mechanical structure. The tool is simple, and the cost of manufacturing and maintenance is low. More important, the all-metal structural design can withstand higher temperatures and high temperature environments such as ultra-deep wells and vertical wells with abnormal geothermal gradients. So, it becomes the only way to achieve active correction during drilling in high temperature and ultra-deep wells [9-11].

Since the high-precision and high-sensitivity electronic control system is abandoned, the deviation control only depends on the characteristic of the heavy block in the mechanical stabilized platform to maintain the low side of the well under the action of gravity, so the gravity sensing mechanism is a key component for sensing well deviation and controlling its actuator to achieve well deviation response. Its performance is very important for mechanical automatic vertical drilling tools. Due to the technical blockade, there are few published researches on mechanical automatic vertical drilling tools [12, 13].

Comeaux et al. [5] discuss a new system which is a purely hydromechanical solution with no electronics. The system automatically initiates corrections at a fraction of a degree deviation from vertical (ideal value $0.2^{\circ}$ ). But, the paper only introduces the design principle and use of the tool and does not specifically discuss the design process and structure.

Ma et al. [6] introduce a new AVDT based on Restoring Braced Structure (EBS). Once well deviation happens, tools take restoring moment to be produced by gravity of offset gravity lock to control the bearing of guide force, so that well straightening is achieved. In the article, the force analysis of the EBS structure and the static design method are provided.

In fact, the working process of MAVDT is dynamic [14-17]. It is generally installed near the drill bit and rotates with the bottom hole assembly during operation and will be affected by axial vibration, lateral vibration, torsional vibration, or other factors, which will affect the response accuracy and stability of the platform. Therefore, the working performance of the tool will eventually change dynamically. Li et al. [9] introduce a kind of MAVDT which has a mechanically sensing mechanism with gravity sensitivity and revealed the dynamics of gravity block under the effect of torsional vibration and found that while the frequency of torsional vibration decreases, a subordinate period gradually appears after the rotation speed value less than $1 \mathrm{rad} / \mathrm{s}$. However, the static design is still used in the design process of the sensing mechanism.

This paper first analyzes the force of the gravity sensing mechanism of the MAVDT. It can be found that the restoring force and friction resistance are the main components that affect the performance of the gravity sensing mechanism; and the restoring force and friction resistance are related to the deviation angle of the gravity sensing mechanism and the coefficient of friction and the force between the disc valves and so on. D'Alembert principle can be used to establish dynamic equations of sensing mechanisms. After that, the acceleration of the gravity sensing mechanism is set to zero first, and the factors affecting the critical state of the gravity sensing mechanism and the key factors affecting the response accuracy and stability of the gravity sensing mechanism are obtained. Finally, multibody dynamics is introduced and numerical analysis is used to analyze the motion characteristics of the gravity sensing mechanism under different initial conditions. Through the introduction of dynamics, the motion model of the gravity sensing mechanism is more reasonable, and the motion characteristics of the gravity sensing mechanism and the responding mechanism of well deviation can be further understood, and it is also beneficial to optimize the structure of the gravity sensing mechanism and further improve the response accuracy and stability of the MAVDT.

\section{Modeling and Methods}

2.1. D'Alembert Principle. D'Alembert's principle [17-21] is a universal method to solve the problem of nonfree particle system dynamics. This method can be used to study the imbalance of dynamics by using statics to study the balance problem, so it is also called the Dynamic Static method. The mathematical expression of D'Alembert is

$$
F_{i}+N_{i}-m_{i} a_{i}=0
$$

where $F_{i}$ is the main force of the particle added to the mass, $N_{i}$ is the restraining force of the limiting particle, and $a_{i}$ is the acceleration of the particle.

D'Alembert suggests that the main force $F_{i}$ can be decomposed into two components, $F_{i a}$ and $F_{i b}$, which is

$$
F_{i}=F_{i a}+F_{i b}
$$

$F_{i a}$ is balanced with the restraining force $N_{i}$, which is

$$
F_{i a}+N_{i}=0
$$

$F_{i b}$ is used to generate inertial force $m_{i} a_{i}$, which is

$$
F_{i b}=-m_{i} a_{i}
$$

Thus, equation (1) is consistent in form with the statics equilibrium equation, and its physical meaning is the main force $F_{i}$, which is received by each particle of the system of particles, and the restraining force $N_{i}$ and inertial force $m_{i} a_{i}$ become the balance force system. In the statics equations, what constitutes the equilibrium force system is the force of external objects on the particle, but, in the mathematical expression of D'Alembert's principle, the inertial force is inherent to the particle and is "virtual force" which is introduced for the convenience of solving the problem, so that dynamic problems can be handled like static balance problems.

Using D'Alembert principle to analyze the movement of the sensing mechanism can realize the unification of the static balance analysis and the dynamic analysis model. When the inertial force is set to zero for analysis, the critical state of the sensing mechanism can be analyzed and then 
introduce inertial force to analyze the change of its motion state.

2.2. Force Analysis and Mathematic Model of the MAVDT's Gravity Sensing Mechanism. As shown in Figure 1, the MAVDT includes gravity heavy block, bearing, outer tube, and disc valve control structure.

The gravity block is the core component for the gravity sensing mechanism sense of well deviation. When the inclination angle exists, the gravity block will produce a restoring torque that always points to the low side of the wellbore. Li et al. [9] proved that, as shown in Figure 2, when the included angle of the heavy block is $\pi$, the largest restoring torque $T_{E}$ produced is as shown in the following equation:

$$
\begin{aligned}
& T_{E}=\frac{2}{3} \rho g l\left(r_{2}^{3}-r_{1}^{3}\right) \sin \beta \sin \phi, \\
& G_{e}=\rho g V=\frac{\pi}{2} \rho g l\left(r_{2}^{2}-r_{1}^{2}\right),
\end{aligned}
$$

wherein $T_{E}$ is the restoring torque, $\mathrm{N} \cdot \mathrm{m} ; G_{e}$ is the gravity of the gravity block, $\mathrm{N} ; r_{1}$ and $r_{2}$ are the inner and outer diameters of the gravity block, respectively, $\mathrm{m} ; \rho$ is the density of the gravity block, $\mathrm{kg} / \mathrm{m}^{3} ; g$ is the acceleration of gravity, the value in this paper is $9.8 \mathrm{~m} / \mathrm{s}^{2}$; and $l$ is the length of the gravity block, $\mathrm{m}$.

The bearings include radial bearings and axial thrust bearings. The radial bearings are used to realize the connection between the gravity block and the outer tube, allowing them to rotate relatively freely under the drivenness of restoring torque. The frictional resistance moment $T_{T B}$ of this part is always opposite to the direction of the relative movement of the gravity heavy block and the outer mandrel. To reduce the influence of the weight of the heavy block on the response accuracy and achieve the tool's high-sensitivity inclination response, rolling ball bearings are usually used as the axial thrust bearing. The frictional resistance torque $T_{A B}$ generated by this bearing to the gravity block during the relative movement is the same as the radial bearing and is always opposite to the relative movement direction of the gravity block and the outer tube. The values of $T_{T B}$ and $T_{A B}$ can be obtained by the following equations:

$$
\begin{aligned}
& T_{T B}=\frac{\pi}{2} \rho g l\left(r_{2}^{2}-r_{1}^{2}\right) \sin \beta \cos \phi \mu_{T B} r_{T B}, \\
& T_{A B}=\frac{\pi}{2} \rho g l\left(r_{2}^{2}-r_{1}^{2}\right) \cos \beta \mu_{A B} r_{A B},
\end{aligned}
$$

wherein $r_{T B}$ is the equivalent friction radius of the radial bearing, $\mathrm{m} ; r_{A B}$ is the equivalent friction radius of the axial thrust bearing, $\mathrm{m} ; \mu_{T B}$ is the friction coefficient of the radial bearing; and $\mu_{A B}$ is the friction coefficient of the axial thrust bearing.

The frictional resistance torque $T_{P}$ between the disc valves is produced by the relative movement between the upper disc valve and lower disc valve. The upper disc valve and the gravity block are connected by a key in the circumferential direction. Under the drive of the gravity block, they are always stable at the bottom of the well deviation or have a tendency to move toward the bottom of the well deviation. The direction of the frictional torque $T_{P}$ of the gravity sensing mechanism movement is opposite to the relative movement direction between the gravity block and the main body, not always the opposite of the movement direction of the gravity block.

In fact, the frictional resistance torque between the disc valves is closely related to the disc valve structure. The disc valve structure used in this study is shown in Figure 3. The theoretical calculation formula for the friction torque between the upper and lower disc valves can be simplified as follows:

$$
T_{P}=F_{P} r_{d b} \mu_{P},
$$

wherein $T_{P}$ is the frictional resistance torque $\mathrm{N} \cdot \mathrm{m} ; \mu_{P}$ is the friction coefficient of the disc valve; $F_{P}$ is the normal force of the fluid on the disc valve and can be calculated by $F_{P}=P S$, where $P$ is the pressure distribution between the upper and lower disc valves and $S$ is the effective area of fluid pressure between the upper and lower disc valves; $r_{d b}$ is the equivalent friction radius of the upper and lower disc valve, $\mathrm{m}$.

As shown in Figure 2, $\beta$ is the angle between the gravity sensing mechanism and the vertical in the initial state; $\phi$ is the angle between the center of gravity of the gravity sensing mechanism and the low side of the wellbore. According to the analysis of the force of the gravity sensing mechanism and D'Alembert principle, the general equation of dynamics of the sensing mechanism in this process can be obtained as follows:

$$
\begin{array}{r}
T_{E}+T_{P}+T_{A B}+T_{T B}-\operatorname{mar}=0, \\
m=\frac{G_{E}}{g},
\end{array}
$$

where $r$ is the distance from the centroid of the platform to the center of rotation, $\mathrm{m} ; a$ is the rotational angular acceleration, $a=\ddot{\phi}$. From equations (5)-(10) and (11), the general equations for the dynamics of the gravity sensing mechanism can be obtained as follows:

$$
\rho \ddot{\phi} \frac{2\left(r_{2}^{3}-r_{1}^{3}\right)}{3}-\rho g l\left(\frac{2}{3}\left(r_{2}^{3}-r_{1}^{3}\right) \sin \beta \sin \phi-\frac{\pi}{2}\left(r_{2}^{2}-r_{1}^{2}\right) \sin \beta \cos \phi \mu_{T B} r_{T B}\right)+\frac{\pi}{2} \rho g l\left(r_{2}^{2}-r_{1}^{2}\right) \cos \beta \mu_{A B} r_{A B}+F_{P} r_{d b} \mu_{P}=0 .
$$




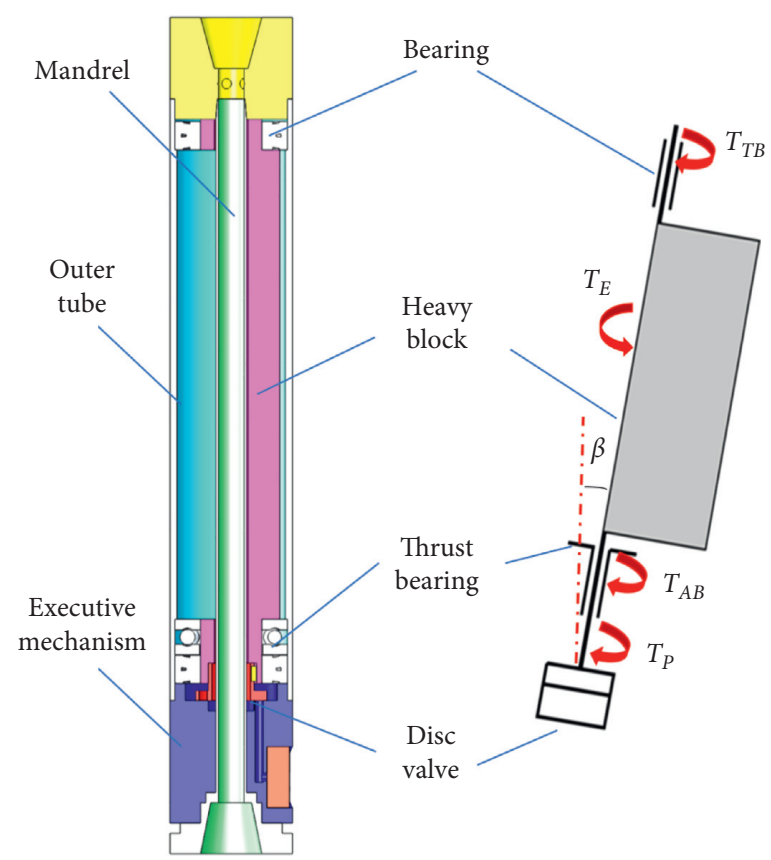

FIGURE 1: The force analysis model of the gravity sensing mechanism of the MAVDT.

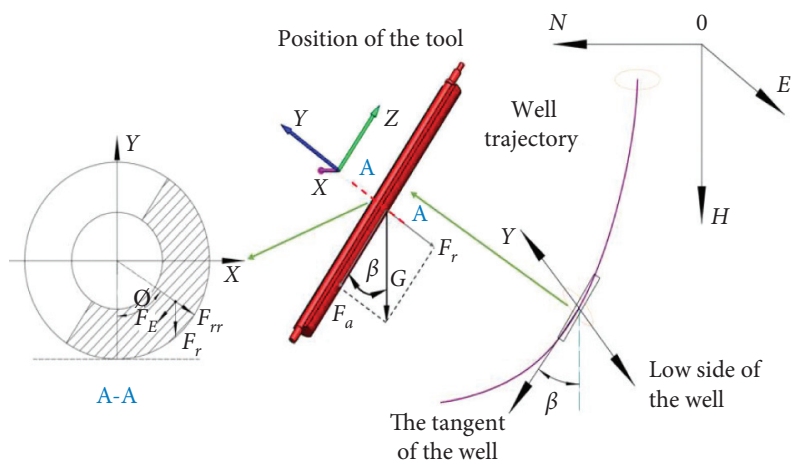

FIGURE 2: Schematic diagram of restoring force calculation of gravity block.

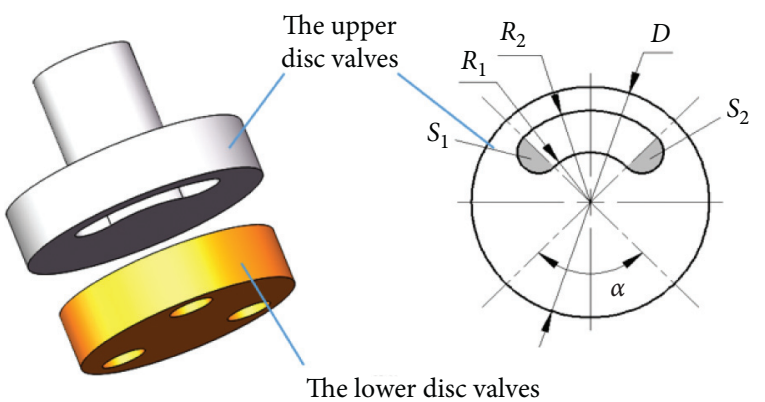

Figure 3: Schematic diagram of disc valve structure [9]. 
The key factors affecting the response accuracy and stability of the sensing mechanism are analyzed based on (12), and the stable position of the sensing mechanism for dynamic analysis is analyzed by using numerical analysis method to deepen the understanding of the stability characteristics of the mechanism.

\section{Design of the Gravity Sensing Mechanism for MAVDT Based on D'Alembert Principle}

For the gravity sensing mechanism, the response accuracy refers to the minimum inclination angle that the platform can achieve response, and the stability refers to the deviation angle between the stable position of the sensing mechanism and the low side of the well inclination under this inclination angle. The gravity sensing mechanism requires a certain degree of response stability within a certain range of well inclination; that is, the sensing mechanism must start to respond when the inclination angle is $\beta$ and the deflection angle is $\phi$, and the acceleration of the platform $a=0$ at this time. And the general dynamics equation can be transformed into the static balance form as follows:

$$
\frac{2}{3} \rho g l\left(r_{2}^{3}-r_{1}^{3}\right) \sin \beta \sin \phi=\frac{\pi}{2} \rho g l\left(r_{2}^{2}-r_{1}^{2}\right) \sin \beta \cos \phi \mu_{T B} r_{T B}+\frac{\pi}{2} \rho g l\left(r_{2}^{2}-r_{1}^{2}\right) \cos \beta \mu_{A B} r_{A B}+F_{P} r_{d b} \mu_{p} .
$$

Analyze the parameters contained in equation (13), limited by the structure, the adjustable range of the inner and outer diameters of the gravity block $r_{1}$ and $r_{2}$, the bearing friction radii $r_{T B}$ and $r_{A B}$, and the disc valve equivalent friction radius $r_{d b}$ which is small and can be regarded as fixed value as the gravity block density $\rho$ and gravitational acceleration $g$. But the friction coefficients $\mu_{T B}, \mu_{A B}$, and $\mu_{P}$ are chosen in accordance with the empirical value in ideal state. The pressure difference $P$ between the two sides of the disc valve that affects the normal force $F_{P}$ of the disc valve is an approximate value obtained according to the tool performance requirements and working principle, and $S$ is area of the upper and lower valve bearing pressure difference.

Based on the previous research and structural design, the theoretical values of the parameters involved in the sensing mechanism are shown in Table 1 in this paper.
Substitute the parameters in Table 1 into the following equation:

$7.93 l \sin \beta \cos \phi+4.1 l \cos \beta+563.46=1705.15 l \sin \beta \sin \phi$.

It can be simplified as follows:

$$
l=\frac{56346}{170515 \sin \beta \sin \phi-793 \sin \beta \cos \phi-410 \cos \beta} \text {. }
$$

Therefore, the length of the gravity sensing mechanism $l$ can be calculated according to the expected response angle $\beta$ and stability angle $\phi$.

In equation (15), derivation $\beta$ and $\phi$ can be used to obtain the change rate of the length $l$ to the response inclination angle $\beta$ and stable deflection angle $\phi$, respectively, as shown in the following equations:

$$
\begin{aligned}
& i_{\beta}=-\frac{56346(170515 \cos \beta \sin \phi-793 \cos \beta \cos \phi+410 \sin \beta)}{(170515 \sin \beta \sin \phi-793 \sin \beta \cos \phi-410 \cos \beta)^{2}}, \\
& i_{\phi}=-\frac{56346(170515 \sin \beta \cos \phi+793 \sin \beta \sin \phi)}{(170515 \sin \beta \sin \phi-793 \sin \beta \cos \phi-410 \cos \beta)^{2}} .
\end{aligned}
$$

Then, the relationship between the length of the gravity block $l_{\phi}$ and the length change rate $i_{\phi}$ with the stability angle $\phi$ under the conditions of different response inclination angles $\beta$ can be obtained as shown in Figure 4. The relationship of response well inclination angle $\beta$ with the length of the gravity block $l_{\beta}$ and the inclination angles $\beta$ under the conditions of different stable angles $\phi$ with the change rate of the length of the gravity block $i_{\beta}$ can be obtained as shown in Figure 5 .

The angle $\phi$ between the planes which is generated by the centroid of the gravity block and the axis of rotation and the low side of the wellbore ranges from 0 to $2 \pi$. When it exceeds $\pi$, the direction of the force is opposite to the set positive direction. Therefore, it is reasonable that the calculated gravity block length becomes a negative value. At this time, only the value and change trend are taken. It can be seen from Figure 4 that when the maximum allowable deflection angle of the gravity block $\phi$ is the same, as the response well deviation angle $\beta$ decreases, that is, the response accuracy requirement increases, the required gravity block length $l_{\phi}$ increases, and the rate of the gravity block length changes corresponding to the deflection angle that gradually increases. When the well deviation response angle $\beta$ is the same, the longer the gravity block, the smaller the deflection angle $\phi$ when the gravity sensing mechanism reaches stability. When the deflection angle is in range of $[0,(\pi / 2)]$ or $[(3 \pi / 2), 2 \pi]$, the length of the gravity block $l_{\phi}$ is negatively correlated with the stable angle $\phi$. And when the deflection angle is in range of $[(\pi / 2),(3 \pi / 2)]$, the length of the gravity 
TABLE 1: The theoretical values of platform design parameters.

\begin{tabular}{lc}
\hline Parameter name & Parameter value \\
\hline Outer diameter $r_{2}(\mathrm{~m})$ & 0.11 \\
Inner diameter $r_{1}(\mathrm{~m})$ & 0.045 \\
Density $\rho\left(\mathrm{kg} / \mathrm{m}^{3}\right)$ & 11000 \\
Gravity acceleration $g\left(\mathrm{~m} / \mathrm{s}^{2}\right)$ & 9.8 \\
Friction radius of thrust bearing $r_{A B}(\mathrm{~m})$ & 0.08375 \\
Friction coefficient of thrust bearing $\mu_{A B}$ & 0.0015 \\
Friction radius of radial bearing $r_{T B}(\mathrm{~m})$ & 0.081 \\
Friction coefficient of radial bearing $\mu_{T B}(\mathrm{~m})$ & 0.0025 \\
Fluid pressure on disc valve $P(\mathrm{MPa})$ & 1 \\
Pressure area of disc valve $S\left(\mathrm{~mm} \mathrm{~m}^{2}\right)$ & 2713 \\
Friction radius of disc valve $r_{P}(\mathrm{~m})$ & 0.054 \\
Friction coefficient of disc valve $\mu_{P}$ & 0.2 \\
\hline
\end{tabular}

block $l$ is positively correlated with the stable angle $\phi$. And the closer the plane formed by the low side of the well inclination and the spin axis, the greater the change rate of the gravity block's length $\dot{i}_{\phi}$. This is because the gravity block has the largest restoring force at the position perpendicular to the low side of the well and the increase in the gravity block length required to overcome the frictional force is the smallest. On the other hand, if the gravity block is required to be stable in the smaller deflection angle range, the length increase required for gravity block length is bigger; and the smaller the stable angle, the greater the change rate of the length of the gravity block required to reduce the stable angle. It also shows that, under the condition of the same well deviation accuracy response, the larger the length of the gravity block, the smaller the angle of the centroid required for the response of the gravity sensing mechanism to deviate from the low side position. This angle can be considered to be the static stability angle, but when the static stability angle is reduced to a certain value, the length of the gravity block required for further improvement is huge. At the same time, it can be found that when the length of the gravity block is constant, if the accuracy requirement of the well deviation response increases, the deflection angle required for stability increases too; that is, the static stability angle gradually increases.

In the design process, it is generally required that the mechanical automatic vertical drilling tool can realize the well deviation response when $\beta \leq(\pi / 36)$. According to the actual situation, it can be slightly increased, but it should not be too large. Therefore, only the well deviation angle $\beta \leq(\pi / 10)$ is analyzed in Figure 5. It can be seen from the figure that when the well inclination angle $\beta$ is constant, the stability angle $\phi$ is negatively correlated with the length $l_{\beta}$ of the gravity block. The smaller the required stability angle, the longer the gravity block required, and the greater the rate of change $i_{\beta}$ of the gravity block length at this time, which means the smaller the required stability angle, the greater the change in the length of the gravity block required to improve the response accuracy; when the deflection angle is constant, as the response well inclination angle decreases, the required gravity block length increases, and the change rate of the length of the gravity block gradually increases; when the length of the gravity block is constant, the smaller the required stability angle, the larger the well inclination angle required to achieve response.

For the gravity block platform, response inclination angle $\beta$ and stability angle $\phi$ are both important performance parameters. In the design process, it is expected that its response accuracy will be relatively high; that is, the response well inclination angle $\beta$ will be relatively small, and, on the other hand, it is also hoped that the stability will be better, which means the deflection angle that can respond is also relatively small, but it can be seen that the response accuracy of the gravity sensing mechanism is negatively related to the allowable stable deflection angle through numerical analysis as shown in Figures 4 and 5. If a parameter is required to increase, it will inevitably need to sacrifice the accuracy of another parameter, and its relationship curve is affected by the other parameters of the gravity sensing mechanism. At the same time, it is also found that, for the gravity sensing mechanism, no matter how long the gravity block is, there is always a minimum limit for the response well deviation angle and the allowable stable deflection angle. The limit value is also related to the selection of the rest of the platform parameters.

As mentioned in the previous article, in the process of platform design, some of the parameter is either fixed or limited by the structure; the adjustable range is narrow. However, some parameter selection is based on experience or coupling design according to other parameter requirements in the design. Whether it has the same effect on the length of the gravity sensing mechanism, and what impacts to the response of the gravity sensing mechanism, requires in-depth analysis to determine the core parameters and the core components that affect the performance of the tool. This is beneficial for clarifying the direction of follow-up work. Therefore, on the basis of determining the relationship between the length of the gravity block and the response angle and the stability angle, this paper analyses the influence of the bearing friction coefficients $\mu_{T B}, \mu_{A B}$, and $\mu_{P}$ and disc valve bearing pressure $P$ on the length of the gravity sensing mechanism one by one. This is necessary to further obtain the optimal relationship between well inclination angle, stable deflection angle, and the length of the gravity sensing mechanism and to further improve the limit response and working ability of the gravity sensing mechanism. 


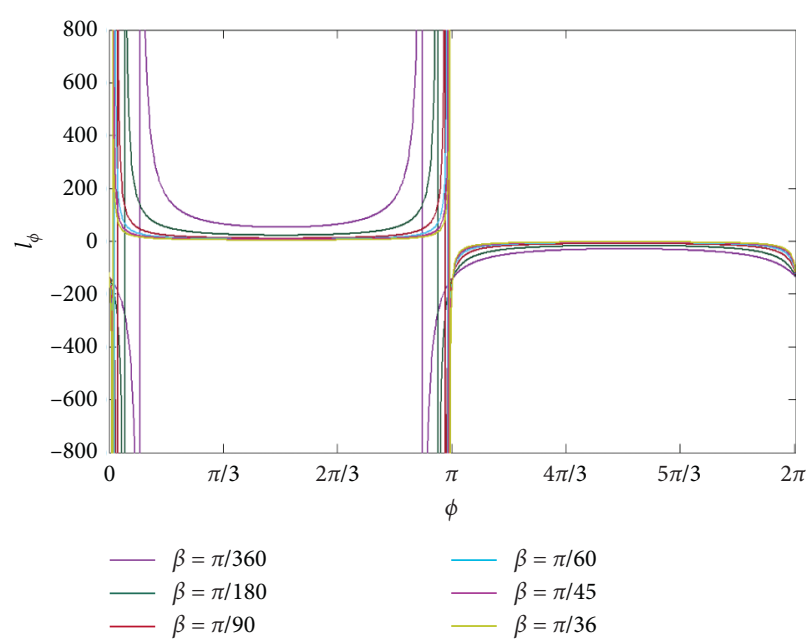

(a)

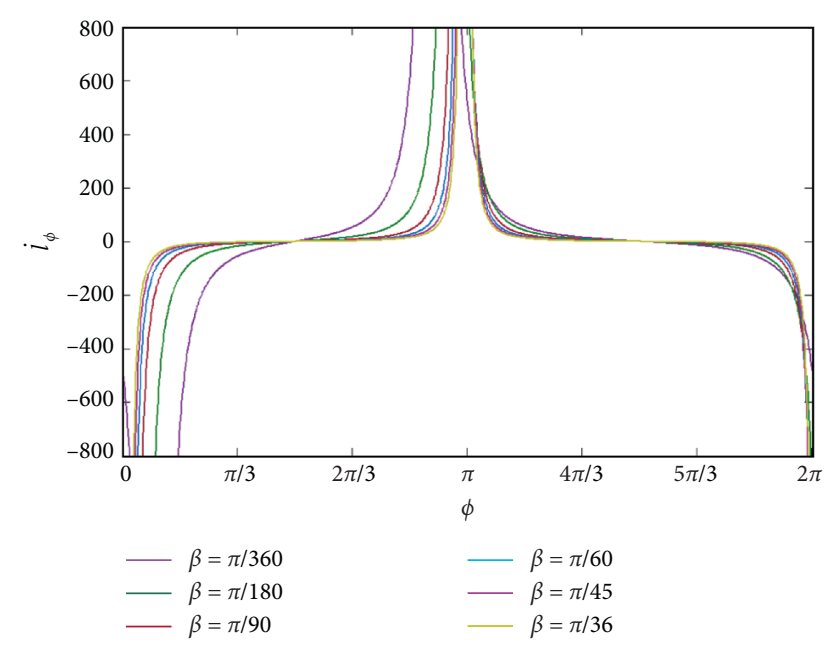

(b)

FIGURE 4: The relationship between the length of the gravity block $l_{\phi}$ and the length change rate $i_{\phi}$ with the stability angle $\phi$ under different well inclination angles $\beta$.

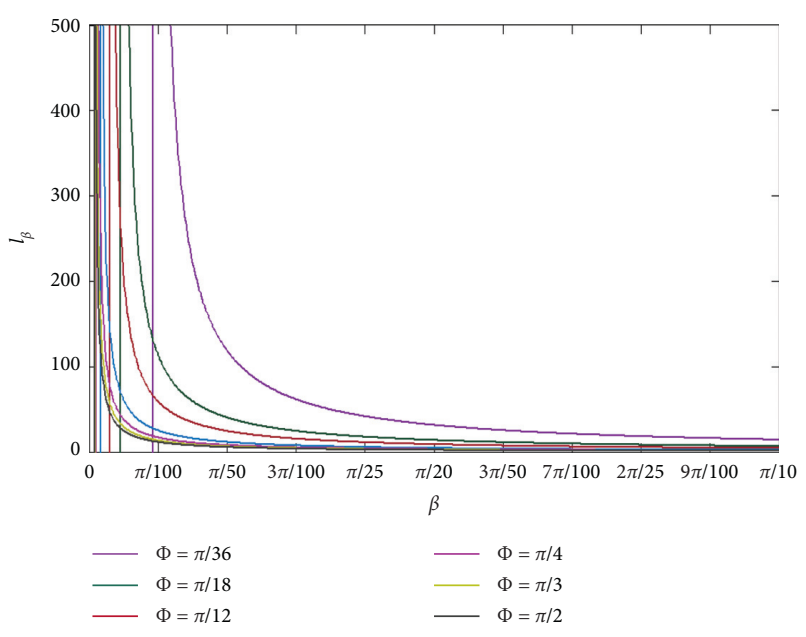

(a)

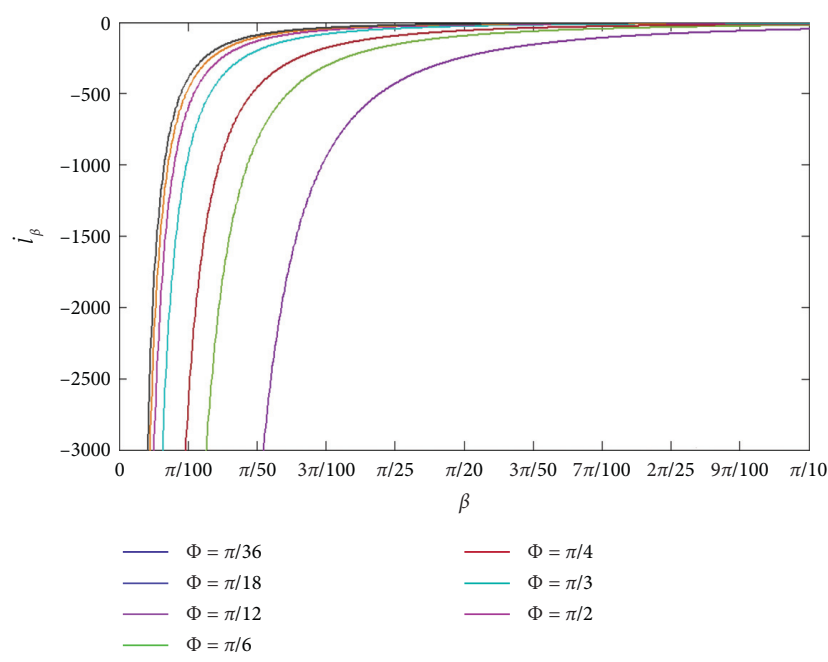

(b)

Figure 5: The relationship between the length of the gravity block $l$ and the length change rate $i_{\beta}$ with the well inclination angles $\beta$ under different stability angles $\phi$.

\section{Analysis of the Key Influencing Factors of the MAVDT'S Gravity Sensing Mechanism's Performance}

4.1. The Influence of Friction Coefficient of Thrust Bearing $\mu_{A B}$ on the Performance of Gravity Sensing Mechanism.
Combining equations (13) and (14) and setting the friction coefficient of the thrust bearing $\mu_{A B}$ as a variable, then the relationship of the gravity block length $l_{\mu_{A B}}$ with the friction coefficient of the thrust bearing $\mu_{A B}$ and the response inclination angle $\beta$ and the stability angle $\phi$ can be obtained as follows:

$$
l_{\mu_{A B}}=\frac{563.46}{1705.15 \sin \beta \sin \phi-7.93 \sin \beta \cos \phi-2732.78 \cos \beta \mu_{A B}} .
$$


Thrust ball bearing is selected in the design process, combined with the empirical value of the friction coefficient; this paper chooses to analyze its influence on the length of the gravity block in the range of $[0.001,0.01]$.

When stability angle $\phi=(\pi / 4)$, the relationship between the length of the gravity block $l_{\beta \mu_{A B}}$ and the friction coefficient of the thrust bearing $\mu_{A B}$ under different well inclination angles $\beta$ can be obtained:

$$
l_{\beta \mu_{A B}}=\frac{28173}{60006 \sin \beta-136639 \mu_{A B} \cos \beta} .
$$

Then, taking the derivative of $\mu_{A B}$, the change rate $\dot{l}_{\beta \mu_{A B}}$ of the length of the gravity block with the change of the friction coefficient of the thrust ball bearing $\mu_{A B}$ under different well inclination angles $\beta$ can be obtained:

$$
i_{\beta \mu_{A B}}=\frac{3849530547 \cos \beta}{\left(60006 \sin \beta-136639 \mu_{A B} \cos \beta\right)^{2}} .
$$

As shown in Figure 6, when the response inclination angle $\beta$ is constant, as the friction coefficient of the thrust bearing $\mu_{A B}$ increases, the length of the gravity block $l_{\beta \mu_{A B}}$ increases too, and the change rate $\dot{l}_{\beta \mu_{A B}}$ also increases. It is particularly important to note that when the corresponding inclination angle $\beta \leq(\pi / 180)$ is required, and other parameters remain unchanged, then there is a maximum choice of friction coefficient of the current thrust bearing within the selection range. For example, when responding to the inclination angle $\beta=(\pi / 360)$, the friction coefficient of the thrust bearing $\mu_{A B}$ is required to be smaller than 0.0038 . Otherwise, no matter how the length of the gravity block increases, the inclination response cannot be achieved. Similarly, when the length of the gravity block is constant, the bigger the friction coefficient of the thrust bearing, the bigger the well inclination angle required to achieve the response, and the bigger the frictional coefficient, the bigger the rate of change in length, indicating that the speed of loss of response accuracy at this time is also increased.

\subsection{The Influence of Friction Coefficient of Radial Bearing $\mu_{T B}$} on the Performance of Gravity Sensing Mechanism. Similarity, combining equations (13) and (14) and setting the friction coefficient of the radial bearing $\mu_{T B}$ as a variable, then the relationship of the gravity block length $l_{\mu_{T B}}$ with the friction coefficient of the radial bearing $\mu_{T B}$ and the response inclination angle $\beta$ and the stability angle $\phi$ can be obtained as shown in the following equation:

$$
l_{\mu_{T B}}=\frac{563.46}{1705.15 \sin \beta \sin \phi-2643 \mu_{T B} \sin \beta \cos \phi-4.1 \cos \beta} .
$$

This paper analyzes the influence of the friction coefficient on the length of the gravity block in the range of [0.001, $0.25]$, and divides it into two intervals of $[0.001,0.25]$ and $[0.001,0.01]$ according to the range of the friction coefficient of rolling bearings and sliding bearings.

When stability angle $\phi=(\pi / 4)$, the relationship between the length of the gravity block $l_{\beta \mu_{T B}}$ and the friction coefficient of the radial bearing $\mu_{T B}$ under different well inclination angles $\beta$ can be obtained:

$$
l_{\beta \mu_{T B}}=\frac{112692}{\left(170515 \sqrt{2}-264300 \sqrt{2} \mu_{T B}\right) \sin \beta-820 \cos \beta} .
$$

And then, taking the derivative of $\mu_{T B}$, the change rate $i_{\beta \mu_{T B}}$ of the length of the gravity block with the change of the friction coefficient of the radial bearing $\mu_{T B}$ under different well inclination angles $\beta$ can be obtained:

$$
i_{\beta \mu_{T B}}=\frac{29784495600 \sqrt{2} \sin \beta}{\left(\left(264300 \sqrt{2} \mu_{T B}-170515 \sqrt{2}\right) \sin \beta+820 \cos \beta\right)^{2}} .
$$

It can be seen from Figure 7 that when the response well inclination angle $\beta$ is constant and the friction coefficient of the radial bearing $\mu_{T B}$ is in the range of 0.001 to 0.25 , as the friction coefficient of the radial bearing $\mu_{T B}$ increases, the length of the gravity block increases, and the rate of change also increases. And with the increase of $\mu_{T B}$, the growth rate of the change rate is also increasing. When the analysis is in the range of 0.001 to 0.01 , it can be found that although the length of the gravity block and the growth rate are increasing with $\mu_{T B}$ increase too, the increment is very small. This shows that when the rolling bearing is selected as the radial bearing, its impact on the response accuracy of the gravity sensing mechanism is small, which can also be confirmed from the growth rate curve as shown in Figure 7(b). At the same time, when the length of the gravity block is constant, the greater the friction coefficient of the thrust bearing $\mu_{T B}$, the bigger the well inclination angle $\beta$ response required, and the greater the $\mu_{T B}$, the greater the rate of change in length $i_{\beta \mu_{T B}}$, indicating that the response accuracy loss rate will also increase at this time. However, it can be found that when the friction coefficient is in the range of 0.001 to 0.01 and the length of the gravity block is fixed, the change of $\mu_{T B}$ has little effect on the response accuracy.

In summary, the friction coefficient of the radial bearing $\mu_{T B}$ has a relatively small effect on the response of the gravity sensing mechanism. In particular, when the rolling bearing is selected, it is generally believed that the friction coefficient will not exceed 0.01 at this time and its impact on the response accuracy is almost ignored. But it should be noted that if the response stability is required to be high, that is, the stability angle $\phi$ is required to be particularly small, the impact will become significant.

4.3. The Effect of Friction Coefficient between Disc Valves $\mu_{P}$ and the Fluid Normal Force $F_{P}$ on the Performance of the Gravity Sensing Mechanism. The friction coefficient between disc valves $\mu_{P}$ and the fluid normal force between disc valves $F_{P}$ have similar effects on the friction resistance torque between the disc valves, so they are put together for comparative analysis.

The same analysis method was used as before; combining equations (13) and (14), and setting the friction coefficient between disc valves $\mu_{P}$ and the fluid normal force between 

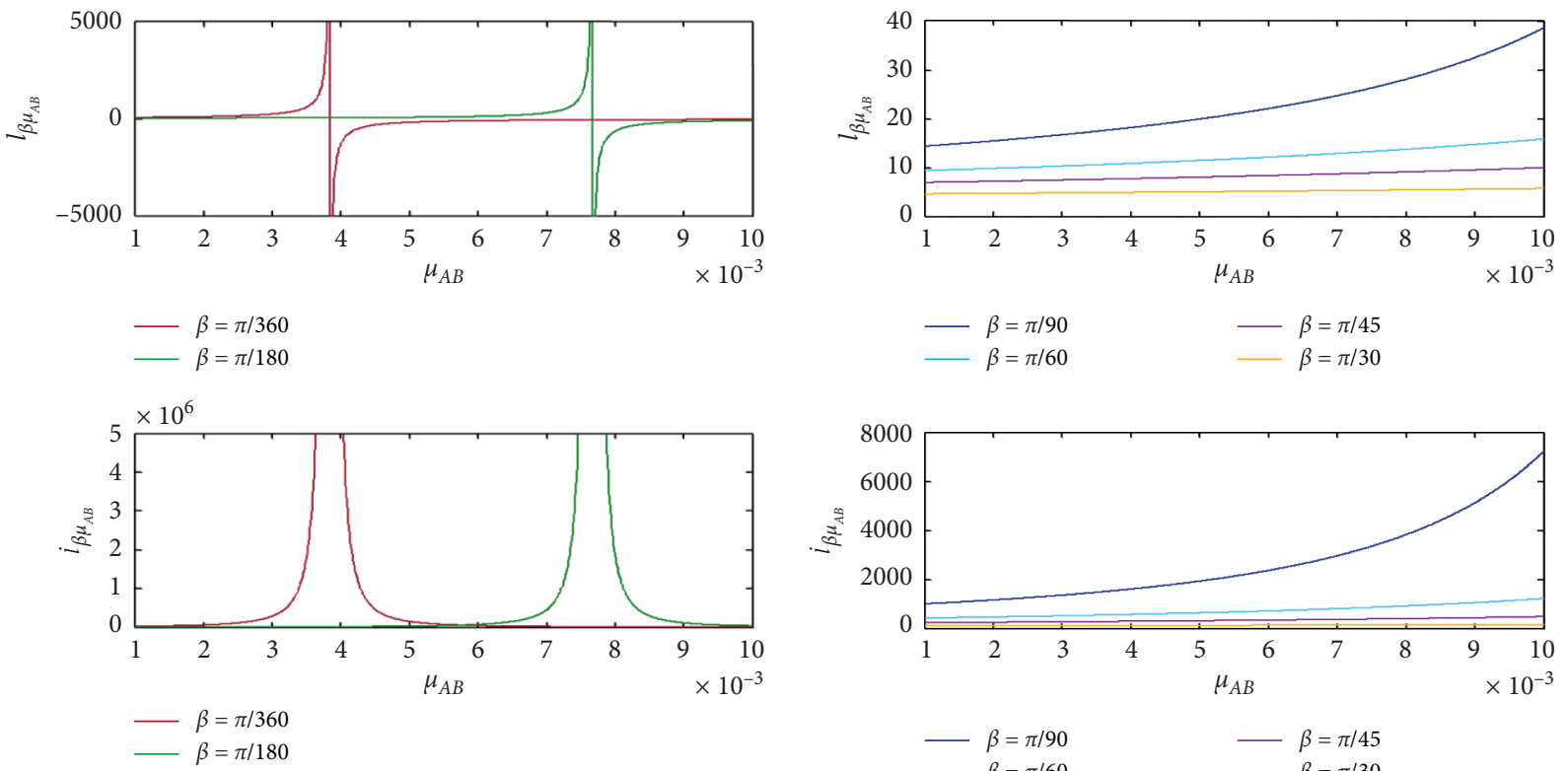

(a)

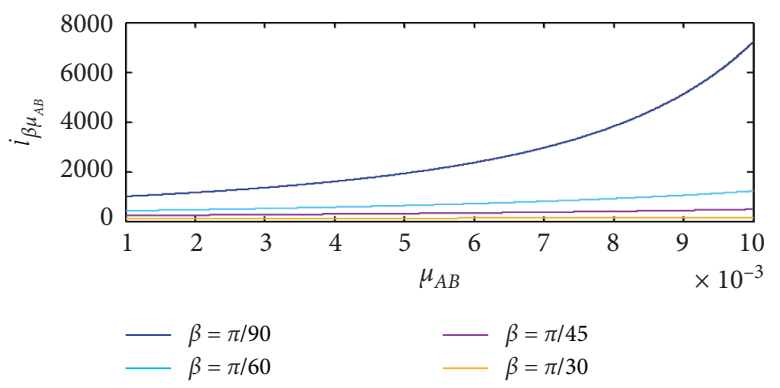

(b)

FIGURE 6: The relationship of the gravity block length $l_{\beta \mu_{A B}}$ and the rate of change $i_{\beta \mu_{A B}}$ with the change of the frictional coefficient $\mu_{A B}$ under the stability angle $\phi=(\pi / 4)$ and different well inclination angles $\beta$.
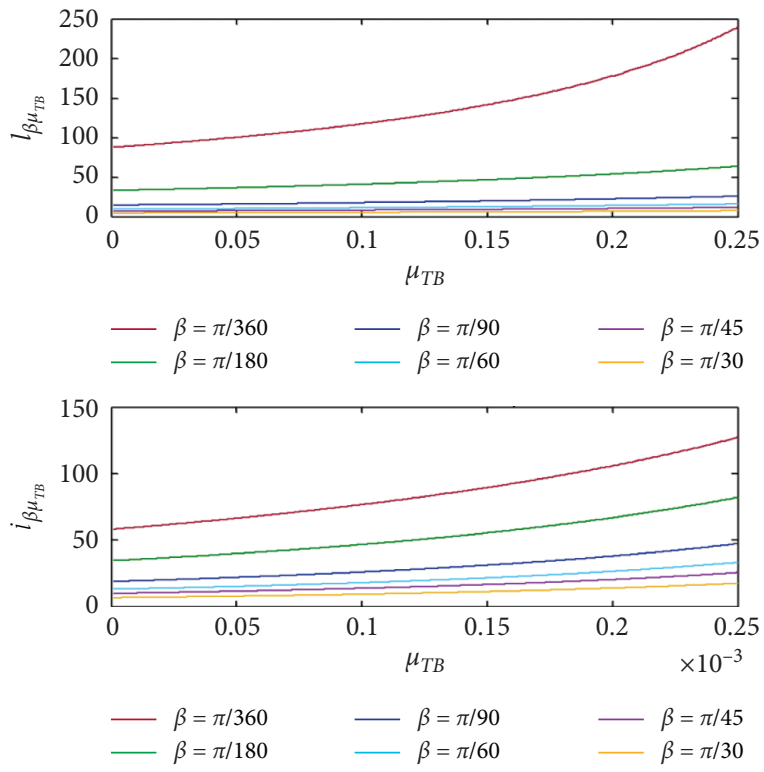

(a)
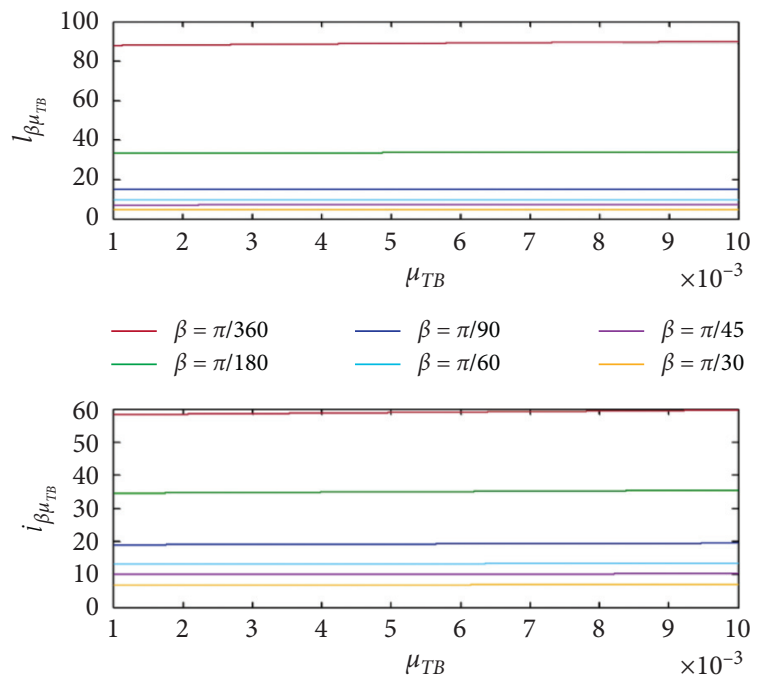

$\beta=\pi / 360$

$-\beta=\pi / 90$

$\beta=\pi / 60$

$-\beta=\pi / 45$

(b)

FIGURE 7: The relationship of the gravity block length $l_{\beta \mu_{T B}}$ and the rate of change $i_{\beta \mu_{T B}}$ with the change of the frictional coefficient $\mu_{T B}$ under the stability angle $\phi=(\pi / 4)$ and different well inclination angles $\beta$. 

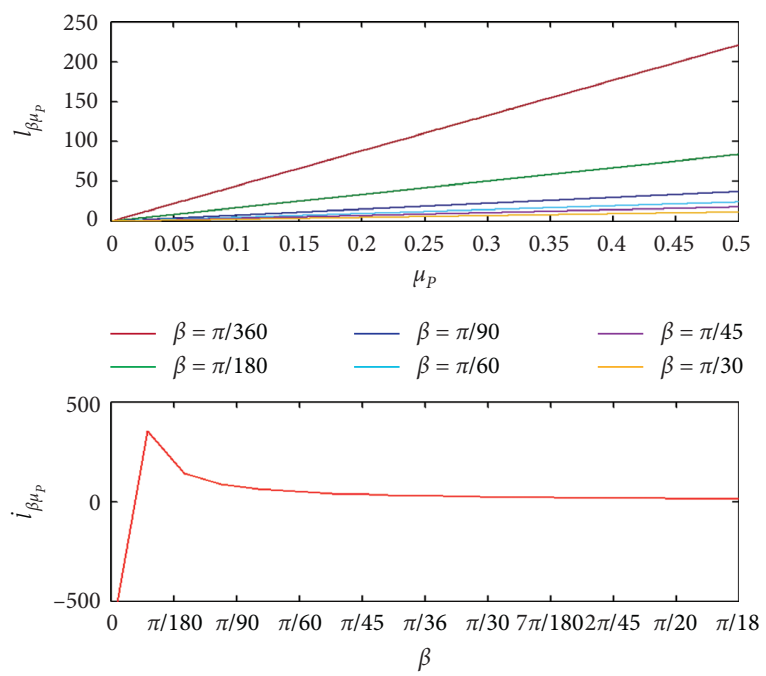

FIGURE 8: The relationship between the length of the gravity block $l_{\beta \mu_{p}}$ and the friction coefficient between the disc valve $\mu_{p}$ under different well inclination angles $\beta$ and the relationship between the change rate of the gravity block length $i_{\beta \mu_{p}}$ and the well inclination angles $\beta$.

disc valves $F_{P}$ as variable, then, the relationship of the gravity block length $l_{\mu_{P}}$ and $l_{F_{P}}$ with the friction coefficient between disc valves $\mu_{P}$ and fluid normal force between disc valves $F_{P}$, respectively, when the response inclination angle is $\beta$ and the stability angle is $\phi$, can be obtained as shown in the following equations:

$$
l_{\mu_{P}}=\frac{2817.3 \mu_{P}}{1705.15 \sin \beta \sin \phi-7.93 \sin \beta \cos \phi-4.1 \cos \beta},
$$

$$
l_{F_{p}}=\frac{0.208 F_{P}}{1705.15 \sin \beta \sin \phi-7.93 \sin \beta \cos \phi-4.1 \cos \beta} \text {. }
$$

When stability angle $\phi=(\pi / 4)$, the relationship of the length of the gravity block $l_{\beta \mu_{P}}$ and $l_{\beta F_{P}}$ with the friction coefficient between disc valves $\mu_{P}$ and the fluid normal force $F_{P}$, respectively, under different well inclination angles $\beta$ can be obtained:

$$
\begin{aligned}
& l_{\beta \mu_{P}}=\frac{28173 \mu_{P}}{12001 \sin \beta-41 \cos \beta}, \\
& l_{\beta F_{P}}=\frac{208 F_{P}}{1200116 \sin \beta-4100 \cos \beta} .
\end{aligned}
$$

And then, take the derivative of $\mu_{P}$ and $F_{P}$, respectively; then, the change ratesi $i_{\beta \mu_{P}}$ and $i_{\beta F_{P}}$ of the length of the gravity block with the change of the friction coefficient between the disc valves $\mu_{P}$ and the fluid normal force $F_{P}$ under different well inclination angles $\beta$ can be obtained:

$$
\begin{aligned}
& i_{\beta \mu_{P}}=\frac{28173}{12001 \sin \beta-41 \cos \beta}, \\
& i_{\beta F_{P}}=\frac{208}{1200116 \sin \beta-4100 \cos \beta} .
\end{aligned}
$$

It can be seen from Figure 8 that when the required response well deviation angle $\beta$ is the same, as the friction coefficient between the disc valves $\mu_{P}$ increases, the length of the gravity block $l_{\beta \mu_{P}}$ increases linearly, and, as the response accuracy requirement increases, that is, the well deviation angle $\beta$ decreases, the growth rate gradually increases. However, it can be found that, with the decrease of the inclination angle $\beta$, the change rate of the gravity block length $i_{\beta \mu_{P}}$ will change abruptly at a certain position and the growth rate will decrease until it becomes a negative value. This is because there is a response limit for the gravity sensing mechanism, and the limit value is related to the friction resistance of other bearings as described in the previous analysis.

It can be seen from Figure 9 that the normal force of the fluid $F_{P}$ acting on the disc valves has the same influence on the length of the gravity block at different inclination angles $\beta$ as the friction coefficients between disc valves $\mu_{P}$ both are linearly related, but the slopes are different.

The friction coefficient $\mu_{P}$ and the fluid normal force $F_{P}$ between the disc valves are two key factors that affect the performance of the gravity sensing mechanism by the disc valve control mechanism. Through the above analysis, it can be found that its influence on the length of the gravity block of the gravity sensing mechanism is linear, and the higher the response accuracy, the greater the impact.

\section{Dynamic Characteristics Analysis of the Gravity Sensing Mechanism}

In the previous article, the critical state where the sensing mechanism starts to realize the response was designed and analyzed. At the same time, the interrelationship of several key parameters affecting the performance of the sensing mechanism including the length of the mechanism, the angle of the well inclination, the angle between the centroid of the 

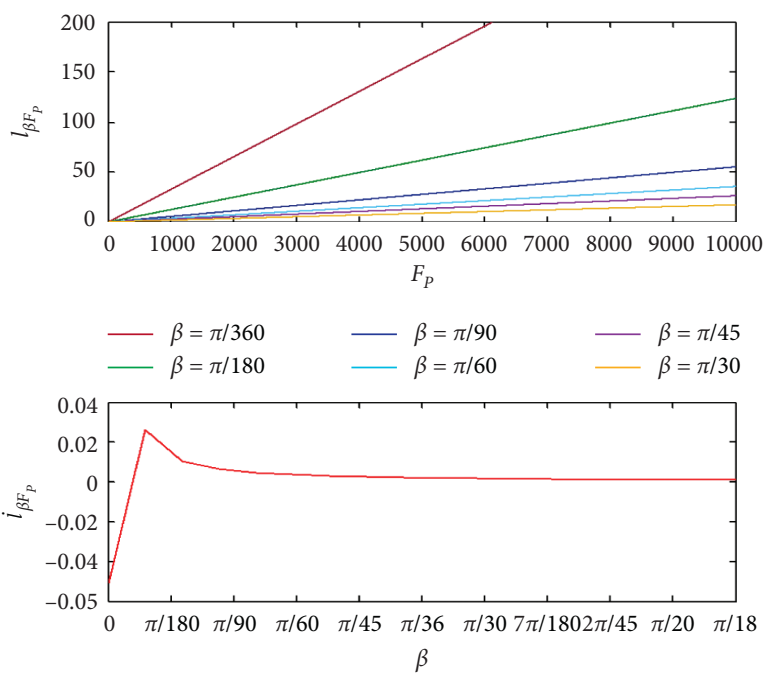

Figure 9: The relationship between the length of the gravity block $l_{\beta F_{p}}$ and the fluid normal force between the disc valve $F_{p}$ under different well inclination angles $\beta$ and the relationship between the change rate of the gravity block length $\dot{i}_{\beta F_{P}}$ and the well inclination angles $\beta$.

mechanism and the low side of the well, the deflection angle, the friction coefficient of the bearing, and the axial fluid force between the upper and lower disc valves was obtained, but this can only obtain the critical conditions for the response of the sensing mechanism and only completed the initial design of the stable platform. In actual use, the working process of the gravity sensing mechanism is a dynamic process. Under a certain well inclination angle, the gravity sensing mechanism will not be just at the critical deflection angle, and the load between the disc valves is not always stable, and when the starting position of the heavy platform is greater than the critical deflection angle, the gravity sensing mechanism response is affected by inertia, and the final stable angle is different from the critical deflection angle. The actual stable position has an important influence on the vertical drilling tool pushing strategy, while the time required for stability will also directly affect the correction accuracy of the tool, so in-depth analysis of the dynamic characteristics of the platform is necessary.

5.1. Dynamic Analysis Model for the Gravity Sensing Mechanism. By analyzing the dynamic general equations of the platform, it can be found that the motion of the platform is a nonlinear dynamic problem and analytical solution is difficult to solve. Therefore, the introduction of numerical analysis has become a more feasible method through simulation. According to the previous platform analysis and design, a three-dimensional solid model as shown in Figure 10 is established, and its main parameter settings are shown in Table 1.

In this research, the length of the gravity block of the platform simulation model is set to $1050 \mathrm{~mm}$. Since the change of the load between the disc valves has an approximate influence on the stable deflection angle and the response inclination angle, the dynamic characteristics of the platform under different well inclination angles and loads between disc valves under deflection angle $\phi=(\pi / 2)$ were analyzed in this research to reduce the variables during the research process.

After the model is established, the constraints should be set for the model firstly before performing simulation analysis. For the mechanical structure, the motion constraints between the components are realized by setting the motion pairs between the components. The motion pairs and related components involved in this example are shown in Table 2.

In addition, the friction relationship needs to be defined for simulating the influence of the frictional damping of each component on the movement of the gravity sensing mechanism. As mentioned above, the friction of the gravity sensing mechanism mainly comes from three sources; the first is the relative friction resistance of the radial bearing and the gravity block component (including the filling material (3), the gravity block (4), and the mounting seat (5)); the second is the relative friction resistance between the axial thrust bearing and the gravity block component; the last is the relative movement between the lower disc valve and the upper disc valve. In the simulation process, the friction between parts is defined by adding contact, and the friction model adopts the Coulomb friction model.

\subsection{The Influence of Well Inclination Angle $\beta$ on the Dynamic} Characteristics of the Gravity Sensing Mechanism. As defined in the previous article, $F_{P}$ is the force between the disc valves, $\phi$ is the angle between the center of mass of the gravity sensing mechanism and the low side of the well inclination, and $\beta$ is the inclination angle. Then, when $F_{P}=100 \mathrm{~N}$ and $\phi=(\pi / 2)$, the oscillation curve of the center of mass of the platform in $Y$ direction under different well inclination angles $\beta$ can be obtained as shown in Figure 11 .

Once the force loaded between the disc valves is constant, the friction resistance between disc valves is fixed too. As shown in Figure 11, if the inclination angle $\beta$ increases, the number of the centers of the mass swing by the lowest 

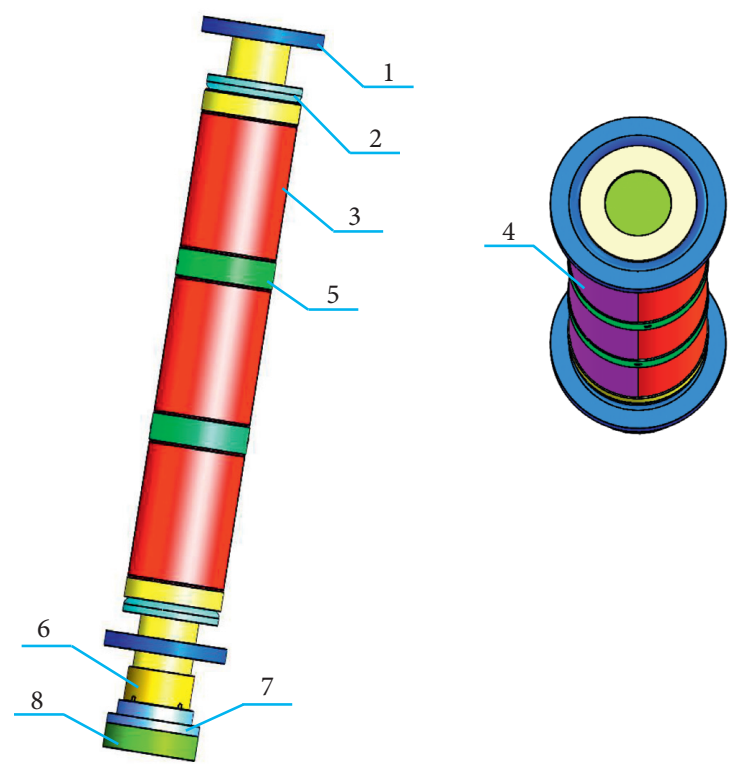

Figure 10: The dynamics simulation model of the gravity sensing mechanism. (1) Radial bearing; (2) axial thrust bearing; (3) filling material; (4) eccentric block; (5) mounting seat; (6) upper disc valve; (7) lower disc valve; (8) tool housing.

TABLe 2: The gravity sensing mechanism dynamics simulation model motion pair settings.

\begin{tabular}{lcc}
\hline $\begin{array}{l}\text { Serial } \\
\text { number }\end{array}$ & Name & Parts involved \\
\hline 1 & Fixed & $1 \& 8 ; 2 \& 8 ; 3 \& 5 ; 4 \& 5 ; 7 \& 8 ; 8 \&$ \\
2 & Cylindrical & ground \\
3 & Mobile & $5 \& 5$ \\
\hline
\end{tabular}

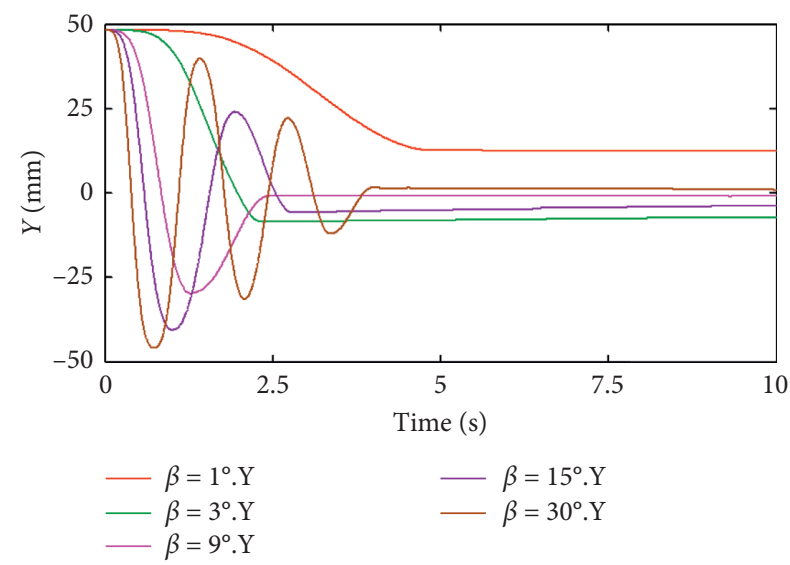

FIgURE 11: The oscillation curve of the center of mass of the platform in $Y$ direction when $F_{P}=100 \mathrm{~N}$ and $\phi=(\pi / 2)$ under different well inclination angles $\beta$.

point of potential energy gradually increases, but the amplitude gradually decreases. As shown in Figure 12, the frequency domain response analysis of the center of mass of the gravity sensing mechanism moving in the $Y$ direction under different inclination angles shows that as the inclination angle $\beta$ increases, the swing periodicity of the gravity sensing mechanism becomes more obvious, which is similar to the change trend of the center of mass motion curve shown in Figure 11. And, with the increase of the inclination angle $\beta$, the frequency of the swing of the gravity sensing mechanism gradually increases.

It can be found through analysis that the stable position and stabilization time of the gravity sensing mechanism are not linearly related to the inclination angle $\beta$, but, in general, as the inclination angle $\beta$ increases, the number of swing cycles of the gravity sensing mechanism increases significantly, and the time required for stabilization gradually increases, while the stable position gradually shifts towards the lowest point of potential energy. This is because the increase of the inclination angle leads to an increase in the circumferential component, and the initial potential energy is also greater. Therefore, under the condition of constant friction, it takes a longer time for energy conversion. As the force in the circumferential direction increases, the deflection angle required to overcome the frictional force at the same inclination angle is also smaller, so its stable position gradually shifts to the direction of the low point of potential energy.

5.3. The Influence of Frictional Resistance on the Dynamic Performance of the Gravity Sensing Mechanism. As mentioned above, the coefficient of friction between the radial bearing and axial bearing and the gravity block in the gravity sensing mechanism, the friction coefficient between the disc valves, and the normal force of the interaction between the disc valves under the action of fluid pressure have an important influence on the design of the gravity sensing mechanism. Meanwhile, the platform will not stabilize at the critical point of response during the process of dynamic action. However, its dynamic stable position has a more direct meaning to the performance of the platform. Therefore, it is necessary to analyze the influence of each key design parameter on the dynamic performance of the platform.

According to the analysis in the previous article, the frictional resistance between the disc valves is an important factor affecting the performance of the gravity sensing mechanism, and it is linearly related to the interaction force between the disc valves and the friction coefficient between the disc valves. This section takes the gravity sensing mechanism in which the stable deflection angle $\phi=(\pi / 2)$ and the well inclination angle $\beta=(\pi / 20)$ are selected as the research object. Let the bearing friction coefficient and the friction coefficient between the disc valves and other parameters be constant and load $10 \mathrm{~N}, 100 \mathrm{~N}, 300 \mathrm{~N}, 500 \mathrm{~N}$, and $650 \mathrm{~N}$ between the upper and lower disc valves, respectively. Then, observe and analyze the swing trajectory of the center of mass in the $Y$ direction as shown in Figure 13.

It can be seen from Figure 13 that as the load between the upper and lower disc valves increases, the number of swing cycles of the gravity sensing mechanism centroid in the $Y$ direction gradually decreases, the stabilization time 


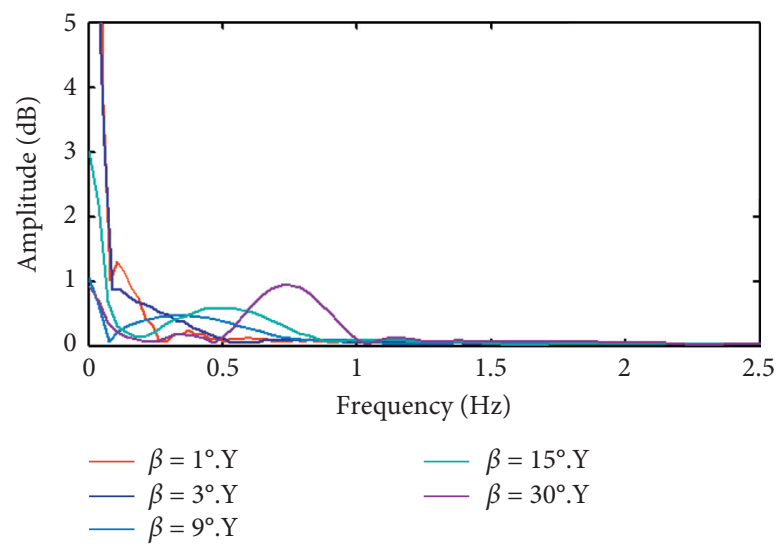

FIGURE 12: The frequency domain response analysis of the centroid of the platform in $Y$ direction when $F_{P}=100 \mathrm{~N}$ and $\phi=(\pi / 2)$ under different well inclination angles $\beta$.

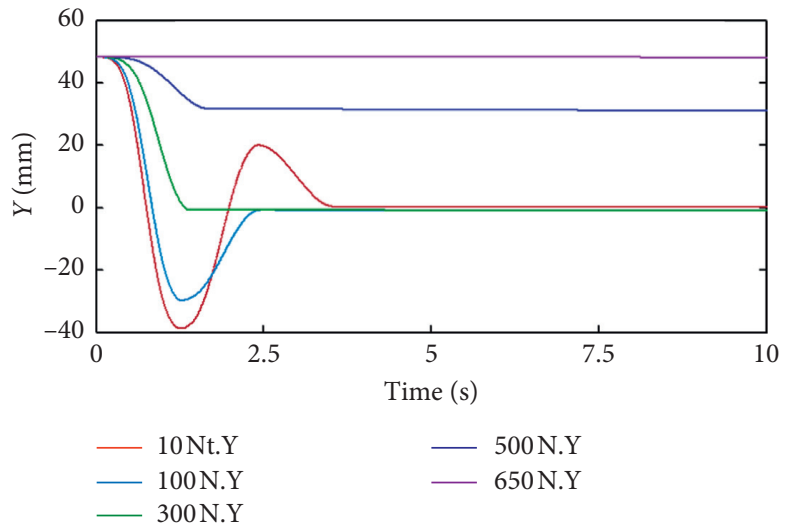

Figure 13: The oscillation curve of the center of mass of the platform in $Y$ direction when $\beta=(\pi / 20)$ and $\phi=(\pi / 2)$ under different loads between the upper and lower disc valves.

gradually shortens, and its stable position gradually deviates from the zero axis (zero point of potential energy) position. After the load increases to $650 \mathrm{~N}$, it is stable at the initial position without swinging.

But, similar to changing the inclination angle, when the loading force decreases, the stable position of the gravity sensing mechanism gradually shifts to the direction of the potential energy zero point. It also can be found that when the loading force reaches $300 \mathrm{~N}$, the centroid also stabilized at the potential energy zero point. Although the stable position gradually deviates from the zero point of potential energy until it fails to respond as the load increases, the time required for stability gradually increases too.

To understand the effect of friction damping on the motion of the gravity sensing mechanism, the number of loads is increased in the analysis, and the swing curves of the gravity sensing mechanism under different loads are shown in Figure 14.

When the interaction load between the upper and lower disc valves is large, the friction force on the sensing mechanism is relatively large, and the acceleration in the circumferential direction of the sensing mechanism is small, so it is not enough to swing the platform to the lower side of the well inclination. The lower side of the well inclination is defined as the zero position of potential energy. At this stage, as the interaction load between the disc valves decreases, the stable position of the sensing mechanism gradually shifts to the direction of the potential energy zero point, and the stabilization time is gradually shortened. This is because as the load decreases, the friction gradually decreases, and the initial acceleration increases, so that the sensing mechanism can reach the stable position faster.

With the further reduction of the interaction load between the upper and lower disc valves, the initial acceleration of the stabilized platform is big enough to cross the zero point of potential energy, but because the friction force is opposite to the restoring torque during the swing to the other side at this time, the direction of acceleration is opposite to the direction of speed and leads the speed to gradually decrease to zero. At this time, the restoring torque value generated at its stable position is not enough to overcome the action of friction and makes it swing again. At this stage, as shown in Figure 14(b), as the load decreases, the stable position gradually deviates from the low side of the well deviation. The time required to reach stability is also shortened as the load decreases too.

With the further decrease of the load, when the sensing mechanism swings to the other side over the zero point of 


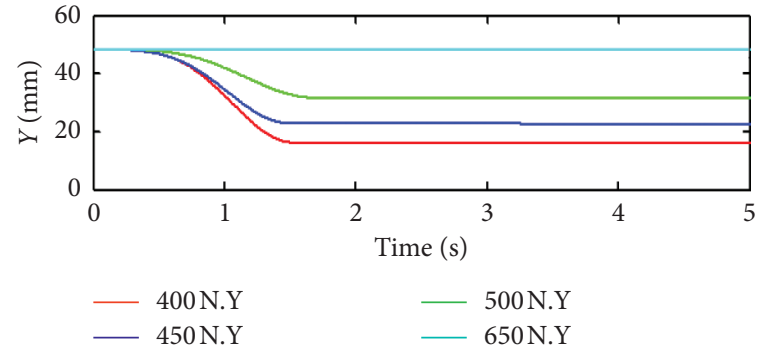

(a)

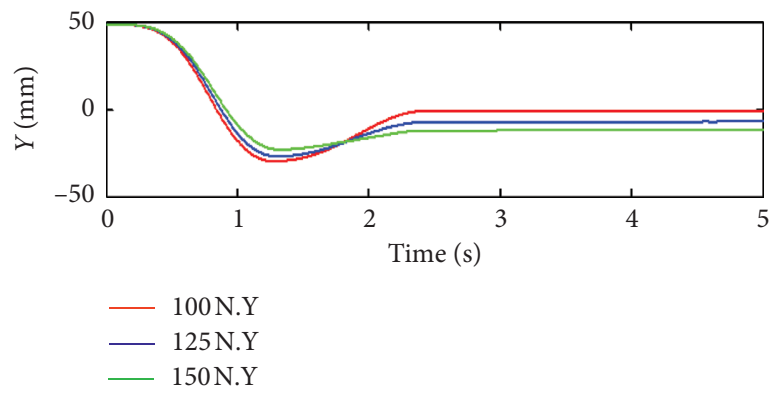

(c)

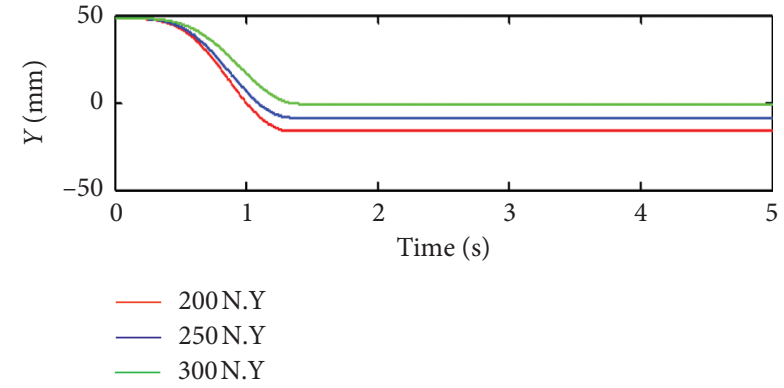

(b)

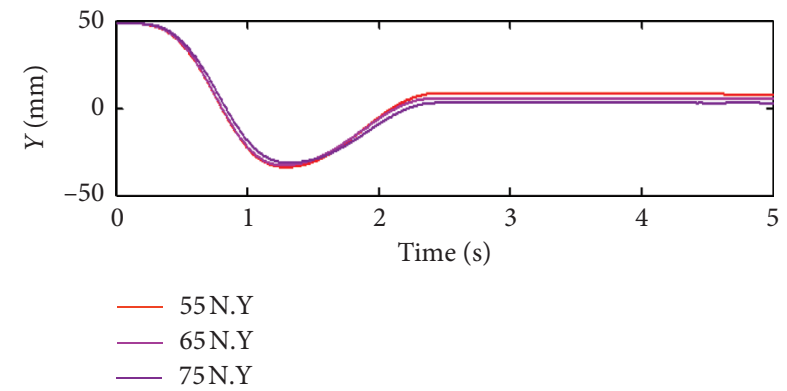

(d)

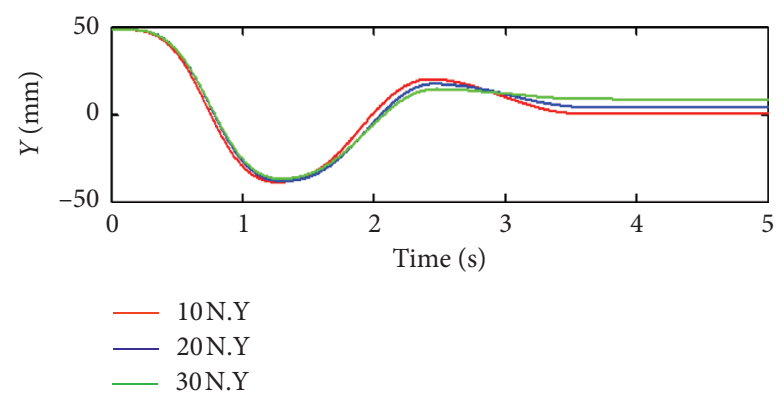

(e)

Figure 14: The oscillation curve of the center of mass of the platform in $Y$ direction when $\beta=(\pi / 20)$ and $\phi=(\pi / 2)$ under different loads between the upper and lower disc valves.

potential energy and the speed drops to zero for the first time, the restoring torque value of this position is bigger than the friction resistance value, and the acceleration value is bigger than zero and points towards the zero point of potential energy. Therefore, the gravity sensing mechanism will not be stable at the first speed zero point position but will swing to the potential energy zero point direction again, as shown in Figure 14(c); at this stage, the stable position is the same as that shown in Figure 14(a). Similarly, as the load decreases, it gradually shifts to the direction of the potential energy zero, and the stabilization time decreases as the load decreases.

Through further analysis of the swing curve of the gravity sensing mechanism as the load decreases, it can be found that the relationship between the stable position of the gravity sensing mechanism and the friction force is related to the status that the gravity sensing mechanism passes through the zero point of potential energy and the zero point of velocity under the action of friction and restoring torque. To be precise, when the load makes the sensing mechanism cannot cross the zero point of potential energy, the stable position of the sensing mechanism gradually shifts to the direction of the potential energy zero as the load decreases. When it is not enough to pass the first speed zero point, the stable position will gradually deviate from the direction of the potential energy zero point as the load decreases. After passing the speed zero point, it will enter the cycle again until it stabilizes at a certain position. But, in general, the stable position gradually shifts to the direction of the potential energy zero as the load decreases, which is also consistent with the previous analysis.

The stability time increases as the load decreases for the gravity sensing mechanism. But because the interval between the zero point of potential energy and the zero point of velocity is crossed, the stabilization time is also shortened as the load decreases.

To analyze the influence of the interaction force between the disc valves on the swing frequency of the gravity sensing mechanism, this paper selects the gravity sensing mechanism at the inclination angle $\beta=(\pi / 6)$ as the research object 


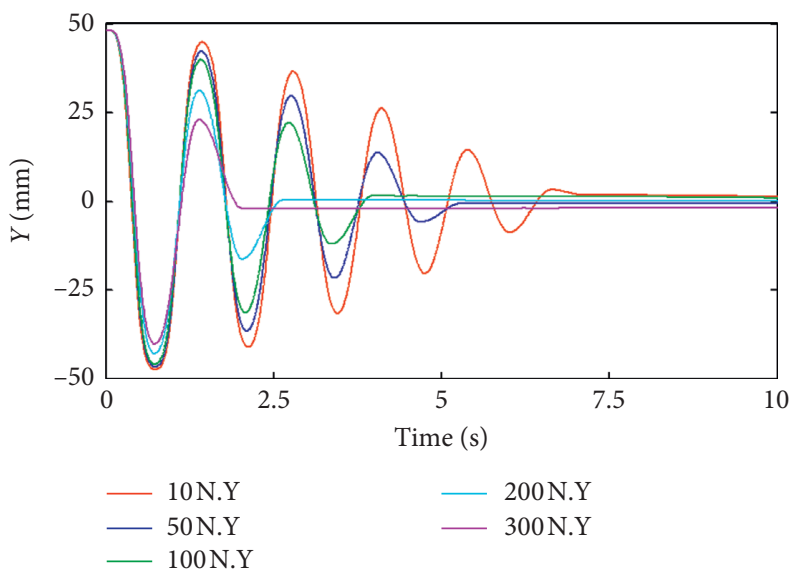

FiguRE 15: The oscillation curve of the centroid of the platform in $Y$ direction when $\beta=(\pi / 6)$ and $\phi=(\pi / 2)$ under different loads between the upper and lower disc valves.

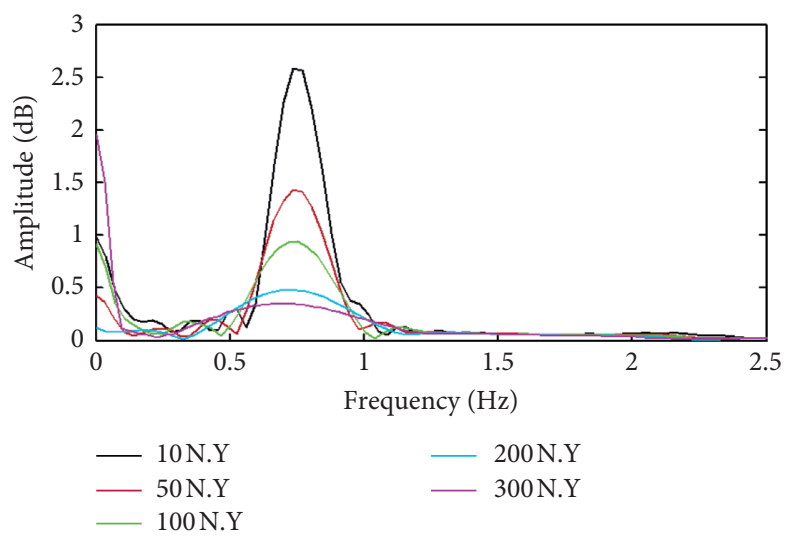

Figure 16: The frequency domain response analysis of the platform centroid in $Y$ direction when $\beta=(\pi / 6)$ and $\phi=(\pi / 2)$ under different loads between the upper and lower disc valves.

and obtains its centroid swing curve under different loads as shown in Figure 15. It can be found that as the load decreases, the number of swing cycles of the sensing mechanism gradually increases, and the stable time gradually increases. The stable position swings near the zero point of potential energy. But when the load is $10 \mathrm{~N}$, it deviates farther from the zero point of potential energy than $300 \mathrm{~N}$, which also proves that the stable position is not directly related to the load.

The frequency response analysis of the swing curve of the gravity sensing mechanism centroid is performed, and the result is shown in Figure 16. It can be seen from the figure that as the load increases, the swing period characteristic of the gravity sensing mechanism is weakened, but the swing frequency remains basically stable.

In fact, the influence of the friction coefficient of the bearing and the friction coefficient between the disc valves on the swing of the gravity sensing mechanism is analyzed, and the result is similar to the effect of the interaction force between the upper and lower disc valves on the swing law of the gravity sensing mechanism; the difference lies in the magnitude of the effect. Among them, the interaction load between the disc valves is often relatively large, and the friction coefficient between the disc valves is much larger than the friction coefficient of the bearing, so the friction coefficient between the disc valves has more significant influence on the sensing mechanism.

\section{Discussion}

In this paper, the dynamic method is introduced in the design process of the MAVDT's gravity sensing mechanism. In the process, there are some new findings:

(1) There are conflicts between the response accuracy and control stability of the sensing mechanism. High response accuracy often means poor control stability. According to the analysis of the impact of each key parameter on the performance of the platform, the impact on the response accuracy and stability is the opposite.

(2) The friction coefficient of the axial thrust bearing of the gravity sensing mechanism has an important influence on the performance of the gravity sensing mechanism. There is a limit value of the allowable friction coefficient for the gravity sensing mechanism 
with certain structural parameters. When the friction coefficient of the thrust bearing exceeds the limit value, the gravity sensing mechanism will not achieve the inclination response at any inclination angle and deflection angle.

(3) The friction coefficient between the disc valves and the force between the disc valves of the sensing mechanism have a linear effect on the performance of the sensing mechanism, and the smaller the deviation angle, the greater the influence coefficient of the force or the friction coefficient between the disc valves on the length of the gravity sensing mechanism.

(4) During the process of dynamic swing, the dynamic stable position of the gravity sensing mechanism is related to the relative relationship between the restoring force of the gravity sensing mechanism and the friction damping. To be precise, it is related to the potential energy zero point and the speed zero point during the gravity sensing mechanism swing process before it reaches the dynamic stable position. When the load makes the sensing mechanism cannot cross the zero point of potential energy, the stable position of the sensing mechanism gradually shifts to the direction of the potential energy zero as the load decreases. When it is not enough to pass the first speed zero point, the stable position will gradually deviate from the direction of the potential energy zero point as the load decreases. After passing the speed zero point, it will enter the cycle again until it stabilizes at a certain position. But, in general, the stable position gradually shifts to the direction of the potential energy zero as the load decreases.

This is beneficial for understanding the dynamic working characteristics of the tool and optimizing the performance of the tool. However, the performance of the tool is not only affected by well deviation angle, position deflection angle, and changes in axial load between disc valves in actual use, but also by bottom hole axial vibration, whirl, stick-slip, and other factors, which will promote the changes of the force on the sensing mechanism during work more complicated, and the dynamic characteristics of the sensing mechanism will inevitably change, which will affect the actual working performance of the MAVDT. Therefore, this should be focused on in the subsequent research work.

\section{Conclusion}

The general dynamic equation of the gravity sensing mechanism is established based on D'Alembert principle by analyzing the force on the gravity sensing mechanism. Due to its complex dynamic equation form and difficulty in solving, this paper takes the critical response position of the sensing mechanism with zero acceleration value as the research object to complete the sensing mechanism preliminary design and analyzed the effect of the force between disc valves on the performance of the sensing mechanism. Based on the analysis of the critical position of the restoring stabilized platform, numerical analysis is used to analyze the influence of the inclination angle and frictional resistance on the dynamic stable position of the gravity sensing mechanism. Some important conclusion can be obtained in this process:

(1) In the actual design process, the requirement of response sensitivity and control stability should be made according to the needs of the project. They have to be balanced.

(2) The friction resistance between disc valves has the greatest influence on the platform performance. The disc valve can be optimized from reducing the friction coefficient between disc valves and the fluid pressure acting on the contact surface.

(3) Reducing friction resistance is beneficial for improving response sensitivity, so friction reduction is an important part in the design of mechanical automatic vertical drilling tools, but its influence on stable position should be considered.

\section{Data Availability}

The data in the tables used to support the findings of this study are included within the article. The data in the figures used to support the findings of this study are available from the corresponding author (hyb@cugb.edu.cn) upon request.

\section{Conflicts of Interest}

The authors declare that they have no conflicts of interest.

\section{Acknowledgments}

This work was supported by the National Key R\&D Program of China (2016YFE0202200), Natural Science Foundation of China (51704264), basic research fund of Chinese Academy of Geological Sciences (JYYWF20180501), and Fundamental Research Funds for the Central Universities (2-9-2018-096).

\section{References}

[1] Q. Xue, R. Wang, L. Huang, F. Sun, and L. Han, "The strapdown automatic vertical drilling system design and field applications," Electronic Journal of Geotechnical Engineering, vol. 17, pp. 3009-3018, 2012.

[2] L. Han, H. Ni, J. Zhao et al., "Development of mechanical tool for automatic vertical drilling," Acta Petrolei Sinica, vol. 29, no. 5, pp. 766-768, 2008, in Chinese.

[3] J. Oppelt, C. Chur, D. Field, and R. Juergens, "New concepts for vertical drilling of boreholes," in Proceedings of the 1991 IADC/SPE Drilling Conference and Exhibition, Amsterdam, Netherlands, March 1991.

[4] W. Wentao, W. Jinquan, W. Xiaotong et al., "Development of full rotation and push-the-bit type automatic vertical drilling tool," China Petroleum Machinery, no. 8, pp. 47-50, 2015.

[5] B. Comeaux, J. Gibb, K. Kirkhope, and P. Shaw, "New automatic vertical drilling system for high temperature, harsh environment and performance drilling applications," in Proceedings of the 2007 Offshore Mediterranean Conference and Exhibition, Ravenna, Italy, March 2007. 
[6] Y. Ma, H. Xia, J. Fu, and P. Yang, "A new design of automatic vertical drilling tool,” Petroleum, vol. 1, no. 3, pp. 264-268, 2015.

[7] A. Ligrone, J. Oppelt, A. Calderoni et al., The Fastest Way to the Bottom: Straight Hole Drilling Device-Drilling Concept, Design Considerations, and Field Experience, Society of Petroleum Engineers, Richardson, TX, USA, 1996.

[8] M. Reich, M. Oesterberg, H. Montes et al., Straight Down to Success: Performance Review of a Vertical Drilling System, Society of Petroleum Engineers, Richardson, TX, USA, 2003.

[9] L. Li, Q. Xue, B. Liu et al., "The dynamics of eccentric block in a fully mechanical vertical drilling tool under the effect of torsional vibration," Advances in Mechanical Engineering, vol. 10, no. 4, 2018.

[10] Y. Shinmoto, Y. Kido, and K. Wada, "Riserless vertical drilling operations using downhole motors in the Nankai-trough seismogenic zone," in Proceedings of the 2012 International Petroleum Technology Conference, Bangkok, Thailand, November 2012.

[11] S. Jones, C. Feddema, J. Castro, and J. Sugiura, "Fully mechanical vertical drilling system delivers RSS performance in vertical drilling applications while providing an economical alternative to conventional rotary steerable systems set-up for vertical hold mode," in Proceedings of the 2016 IADC/SPE Drilling Conference and Exhibition, Fort Worth, TX, USA, March 2016.

[12] T. Ma, P. Chen, and J. Zhao, "Overview on vertical and directional drilling technologies for the exploration and exploitation of deep petroleum resources," Geomechanics and Geophysics for Geo-Energy and Geo-Resources, vol. 2, pp. 365-395, 2016.

[13] D. X. Jiang, J. Zhou, and F. U. Xin-Sheng, "Stability controlling model of built in restoring directional controller," Journal of Xian Petroleum Institute, vol. 15, no. 1, pp. 44-49, 2000.

[14] Q. Xue, H. Leung, R. Wang et al., "The chaotic dynamics of drilling," Nonlinear Dynamics, vol. 83, no. 4, 2015.

[15] D. X. Wang, H. Liu, and S. Han, "Deep rock mechanics and deep or ultra-deep well drilling technology," Drilling and Production Technology, vol. 29, no. 3, pp. 6-10, 2006.

[16] Y. Chunxu, H. Laiju, B. U. Yuhuan et al., "2-D model of BHA mechanical analysis for automatic vertical drilling," Oil Drilling \& Production Technology, vol. 32, no. 1, pp. 26-30, 2010.

[17] H. Liu, T. Ma, P. Chen, X. Wang, and X. Wang, "Mechanical behaviors of bottom hole assembly with bent-housing positive displacement motor under rotary drilling," Arabian Journal for Science and Engineering, vol. 44, pp. 6029-6043, 2018.

[18] B. Vujanovic, "Conservation laws of dynamical systems via D'Alembert's principle," International Journal of Non-Linear Mechanics, vol. 13, no. 3, pp. 185-197, 1978.

[19] J. H. Hubbard and H. B. West, Differential Equations: A Dynamical Systems Approach, Springer-Verlag, Berlin, Germany, 1995.

[20] E. O. Doebelin, System Dynamics: Modeling, Analysis, Simulation, Design, Marcel Dekker, New York, NY, USA, 1998.

[21] B. Qiu, Analytical Mechanics, China Railway Publishing House, Beijing, China, 1998. 